\title{
An International Medical Graduate's Experience of Postgraduate Training in the United States
}

Juan J. Delgado-Hurtado.?

\section{The Experience}

About the Author: Dr. Delgado-Hurtado is a firstyear Internal Medicine resident at Geisinger Medical Center (Danville, PA. United States). He completed his undergraduate medical education in Guatemala (Universidad Francisco Marroquin).
Submission: Apr 7, 2015 Acceptance: Jul 20, 2015 Publication: Aug 14, 2015
Every year many International Medical Graduates (IMGs) come to the United States to do their medical training. According to the 2014 Main Residency Match Report, 6,355 IMGs (majority of them non-U.S. Citizen IMGs) matched into a program. ${ }^{7}$ Although starting the residency is difficult for most interns, for IMGs it is particularly challenging because of differences in culture, prior education, and the health care system the trainee has been exposed to. ${ }^{2}$ For many, there are language and communication barriers too. ${ }^{3}$

In 2014, after completing medical school in Guatemala, I was accepted to start my training in the United States. Starting my residency in the U.S. was challenging. Most of the clerkships I did back in Guatemala were in public hospitals that had very limited resources and technology. The hospital system where I am practicing seemed complex, more robust, and certainly had more resources. Having been exposed to the health system in a lower-middle income country, it is only natural that I tend to compare my prior hospital experiences as a medical student with my training in the U.S. There are many differences between both medical systems; however, I will discuss some that have captured my attention and might be of interest to students. I have grouped them into five categories: the health care systems in general, technology and patient care, teamwork, patient population, and research. I will end up by sharing my thoughts regarding the different health care systems and giving recommendations for future IMGs.

I will start by briefly comparing the health care systems in general. Although there are many health indicators that can be contrasted, the following are given as an example. According to the World Health Organization, the total expenditure on heath is $6.7 \%$ of GDP in Guatemala, compared to $17.9 \%$ in the United States (Available from: http://www.who.int/countries/usa/ en/, updated 2013; cited 2015 Jun 3). ${ }^{4}$ According to the Living Standards Measurement Survey, only $15.1 \%$ of the Guatemalan population have social security or private health insurance. ${ }^{5}$ Life expectancy for Guatemalans is 68 and 75 years for males and females, respectively, compared to 76 and 81 years in the United States. The probability of dying between 15 and 60 years of age is twice as high for persons in my country compared to the United States (Available from: http://www.who.int/countries/usa/en/, updated 2013; cited 2015 Jun 3). ${ }^{4}$

Technology and patient care is very different. For example, electronic medical records or electronic imaging was not available in Guatemala. Diagnosing diseases was a difficult task; often, we did not have the tests or technology to confirm the diagnosis and had to rely on our bedside assessment much more. Sometimes due to high bed occupancy in the intensive care unit, patients who were unstable had to be admitted to regular wards, with fewer nurses and less monitoring. A patient in the U.S. can be discharged to many places - skilled nursing facilities, rehabilitation, and long term facilities; while in Guatemala, similar patients were frequently discharged to their homes as these other options are almost non-existent. Often, this represented a large burden for the family who had to make adjustments to provide comfort for the sick.

Multidisciplinary team work is now common in U.S. hospitals and less common in my home country. In Guatemala, health care professionals are not as specialized and we did not round with a pharmacist, finance, and ancillary staff as we do here. We ordered all of the medications ourselves and did not have them verified by the pharmacy. The interns drew blood, placed urethral catheters, drew arterial gases, intubated patients, and often transported patients for imaging procedures. Having done these procedures myself has been useful to me here in the U.S., as I have helped ancillary staff with them when needed.

The patient populations I have treated here belong to a different culture, tend to be older with prior health care exposure, and have many previous diagnoses. Before coming here, I had plenty of experience treating patients with infectious diseases such as malaria, dengue, HIV, and cardiovascular diseases, but less experience in treating patients with diseases such as Crohn's, ulcerative colitis, and chronic obstructive pulmonary disease; I had no experience treating patients with Lyme disease. Many of my patients in Guatemala did not have or know their previous diagnosis and were taking fewer medications than patients here in the U.S. Unfortunately poverty is a common

${ }^{1}$ Geisinger Medical Center, Danville, PA. United States. 


\section{Experience}

risk factor for many of the diseases with high incidence and prevalence in low- and middle-income countries.

The amount of research done is considerably more in the U.S. than in Guatemala. Data and resources that support and encourage research are widely available in the U.S. Low- and middle-income countries have plenty of information and experiences to share, but unfortunately research is often not carried out because of lack of motivation, information, training, resources, and time. An interesting recent development involves scientists from high-income countries who support, mentor, and collaborate with researchers from low- and middle-income countries.

To conclude, I believe many of the dissimilarities described above depend on resources and development. Furthermore, I am training at an institution nationally recognized for innovation and high quality care. I believe health systems in high-income and low- to middle-income countries have advantages and disadvantages. They adapt to a reality and to a budget. It is no secret that the health care in the U.S. is expensive and that many changes must occur to make it universally available. There must be plenty of diseases that are well-managed in low- and middle-income countries, with fewer resources and lower costs than in the U.S. but with similar high quality care. Low- and middle-income countries should use the experience of high-income nations to locally implement policies, technology, promote research, use medical guidelines and literature, and endorse ancillary care careers.

After a few months of training in my residency program, I feel more comfortable with the U.S. health system than when I started. I have realized that some things are the same regardless of where you practice. The qualities of great physicians are appreciated everywhere: compassion, empathy, understanding, good communication, and support. These qualities should prevail no matter where you are practicing. I have met great physicians here and in my country of origin.

International Medical Graduates who did medical school in another system might have noticed some of the differences that I have found and should remember it takes some time to feel comfortable training in another system. I agree with the literature that suggests that IMGs bring valuable skills as a result of training elsewhere.? We have been exposed to a diverse epidemiology and sociocultural context, and should use this prior experience to provide high quality care and innovation. We should be proud of having trained in another country and remember that even if the system is different, the human essence in becoming a great physician is the same, and that is what is most valued by our patients.

\section{References}

1. National Resident Matching Program. Results and Data: 2014 Main Residency Match ${ }^{\circledR}$. Washington, DC: National Resident Matching Program; 2014. 2. Saeed $\mathrm{F}$, Majeed MH, Kousar N. Easing international medical graduates entry into US training. J Grad Med Educ. 2011 Jun;3(2):269.

3. Jain P, Krieger JL. Moving beyond the language barrier: the communication strategies used by international medical graduates in intercultural medical encounters. Patient Educ Couns. 2011 Jul;84 (1):98-104.

4. World Health Organization. Country Cooperation Strategy at a glance: Guatemala. Geneva: World Health Organization; 2014.

5. Mahal A, Bowser D, Mitchell A. USAID/Dialogue for social investment in Guatemala. Health financing in Guatemala: a situation analysis and lessons from four developing countries. Guatemala: United States Agency for International Development (USAID); 2009.

6. Chen PG, Nunez-Smith M, Bernheim SM, Berg D, Gozu A, Curry LA. Professional experiences of international medical graduates practicing primary care in the United States. J Gen Intern Med. 2010 Sep;25(9):947-53.

\section{Acknowledgments \\ I thank Matt Hodgkinson for reviewing the first draft of this manuscript.}

Conflict of Interest Statement at Funding

The author has no funding, financial relationships or conflicts of interest to disclose.

\section{Author Contributions}

Conception and design the work/idea, Write the manuscript, Critical revision of the manuscript, Approval of the final version: JJDH.

Cite as:

Delgado-Hurtado JJ. An International Medical Graduate's Experience of Postgraduate Training in the United States. Int J Med Students. 2015 Sep-Dec;3(3):165-6. 